Submitted to:

1999 Particle Accelerator Conference

BNL 65999

- March 29. - April 2, 1999

New York City, New York

\title{
TRANSVERSE PHASE SPACE PAINTING FOR SNS ACCUMULATOR RING INJECTION*
}

\author{
J. Beebe-Wang, Y. Y. Lee, D. Raparia, J. Wei \\ Brookhaven National Laboratory, Upton, NY 11973
}

\begin{abstract}
The result of investigation and comparison of a series of transverse phase space painting schemes for the injection of SNS accumulator ring [1] is reported. In this computer simulation study, the focus is on the creation of closed orbit bumps that give desired distributions at the target. Space charge effects such as tune shift, emittance growth and beam losses are considered. The results of pseudo end-to-end simulations from the injection to the target through the accumulator ring and Ring to Target Beam Transfer (RTBT) system [2] are presented and discussed.
\end{abstract}

\section{INTRODUCTION}

At Brookhaven National Laboratory work is in progress for the design and construction of SNS accumulator ring system [1]. The system consists of a $1 \mathrm{MW}$, expandable to 2MW, accumulator ring [3] and two transfer lines. HighEnergy Beam Transport line (HEBT) [4] brings beam from the end of linac to the injection bump of the ring, and Ring to Target Beam Transport line (RTBT) [2] brings beam from the extraction kicker of the ring to the target.

At the target, the proton beam must meet stringent specifications, as listed in Table 1, in consideration of the stress and lifetime of the target. The proton distribution at the target is crucially determined by the beam distribution in the ring. Furthermore, a proper proton distribution in the ring is critically dependent on the 6-dimensional phase space injection/stacking method. Therefore, in order to obtain the desired proton distribution at the target, one must create a set of suitable closed orbit bumps at the injection that provide a proper phase space painting.

In the current $\mathrm{H}$ charge exchange injection design [5], the horizontal and vertical bumps are formed by sets of four pulsed dipoles. The strengths of the dipoles, in each direction, can be programmed as functions of time during the injection to provide phase space painting. As a part of design study, we ask: (1) can the bumps, as in the current design, satisfy the beam requirements at the target? (2) if can, what are the prescriptions of the bumps as functions of time during the injection? (3) are these prescriptions technically achievable? To answer these questions, we investigated and compared a series of transverse phase space painting schemes by realistic computer simulations.

\footnotetext{
Work performed under the auspices of the U. S. Department of Energy.
}

Table 1 Beam requirements at the target [6]

\begin{tabular}{|l|c|}
\hline Beam horizontal dimension & $200 \mathrm{~mm}$ \\
\hline Beam vertical dimension & $70 \mathrm{~mm}$ \\
\hline $\begin{array}{l}\text { Time-averaged beam current } \\
\text { density over beam footprint }\end{array}$ & $\leq 0.091 \mathrm{~A} / \mathrm{m}^{2}$ \\
\hline $\begin{array}{l}\text { Beam power within target } \\
\text { and outside nominal spot }\end{array}$ & $<5 \%$ \\
\hline $\begin{array}{l}\text { Peak time-averaged beam } \\
\text { current density over } 1 \mathrm{~cm}^{2}\end{array}$ & $\leq 0.182 \mathrm{~A} / \mathrm{m}^{2}$ \\
\hline Peak 1-pulse density over $1 \mathrm{~cm}^{2}$ & $1.89 \times 10^{16}$ proton $/ \mathrm{m}^{2}$ \\
\hline
\end{tabular}

\section{COMPUTER SIMULATIONS}

Three computer simulation codes are used in this study: ACCSIM [7] and SIMPSONS [8] for beam tracking in the accumulator ring, and PARMIIA [9] for the RTBT line. Both ACCSIM and SIMPSONS are capable of tracking a large number of macro-particles through the ring lattice, in the presence of space charge and beam to wall interactions, in full 6-dimensions. In addition to the common features, ACCSIM provides wide range of injection simulation options such as foil specifications, 6$D$ phase space painting and injected particle distributions. On the other hand, SIMPSONS is a powerful tool for extensive study of space charge effect. So, in this study, phase space painting is investigated with ACCSIM and space charge effect is done with SIMPSONS. Presently, efforts are been made [10] in BNL to integrate various codes onto a common platform that accommodates 6-D painting and space charge effects together with magnet field error and misalignment analysis, magnetic fringe field mapping, and beam collimation.

All the physical quantities used in the simulations are chosen to be as close as possible to the specifications in the current dcsign. The lattice functions and other salient parameters used in the simulations are listed in Table 2.

Table 2 Design parameters used in the simulation study

\begin{tabular}{|l|c|}
\hline Beam Kinetic Energy & $1 \mathrm{GeV}$ \\
\hline Beam Average Power & $1.0-2.0 \mathrm{MW}$ \\
\hline Proton Revolution Period & $0.8413 \mu \mathrm{sec}$ \\
\hline Ring Circumference & $220.688 \mathrm{~m}$ \\
\hline Number of Turns Injected & 1225 \\
\hline Beam Emittance $\varepsilon_{x y}$ & $120 \pi \mathrm{mm}-\mathrm{mr}$ \\
\hline Tunes $v_{x} / v_{y}$ & $5.82 / 5.80$ \\
\hline Max. $\beta_{x} / \max . \beta_{y}$ & $19.2 / 19.2 \mathrm{~m}$ \\
\hline Dispersion $X_{p}(\mathrm{max} / \mathrm{min})$ & $4.1 / 0.0 \mathrm{~m}$ \\
\hline
\end{tabular}




\section{PHASE SPACE PAINTING}

Since the beam is injected into the ring at a dispersion-free region [5], beam phase-space painting in the transverse direction is conveniently de-coupled from the longitudinal beam manipulation [11]. Furthermore, painting in the horizontal and vertical direction can be adjusted independently. The injection system is designed to accommodate both $x-y$ correlated and $x-y$ anti-correlated painting schemes, illustrated in Fig. 1.

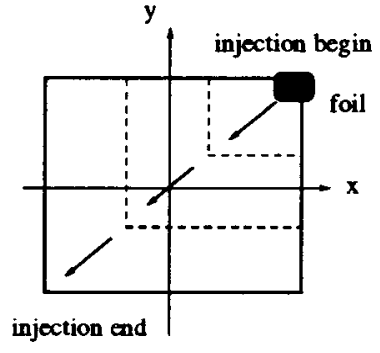

(a)

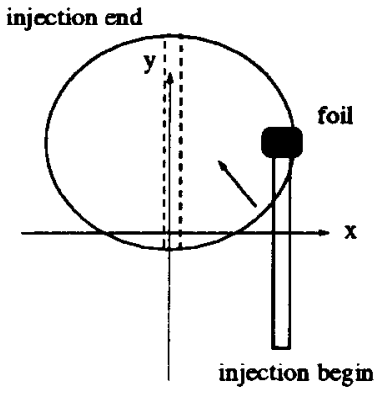

(b)
Fig. 1 Basic painting scenarios. (a) $x-y$ correlated painting, (b) $x-y$ anti-correlated painting.

The most easily achievable bumps are the ones moving the closed orbit monotonically as an exponential function of time with a reasonably long time constant. With a $x-y$ correlated bump setting, as shown in Fig. 2, phase spaces in both dimensions are painted from small to large emittances. Ideally, the resulting rectangular transverse profile, Fig. 3, can easily meet the target requirements.
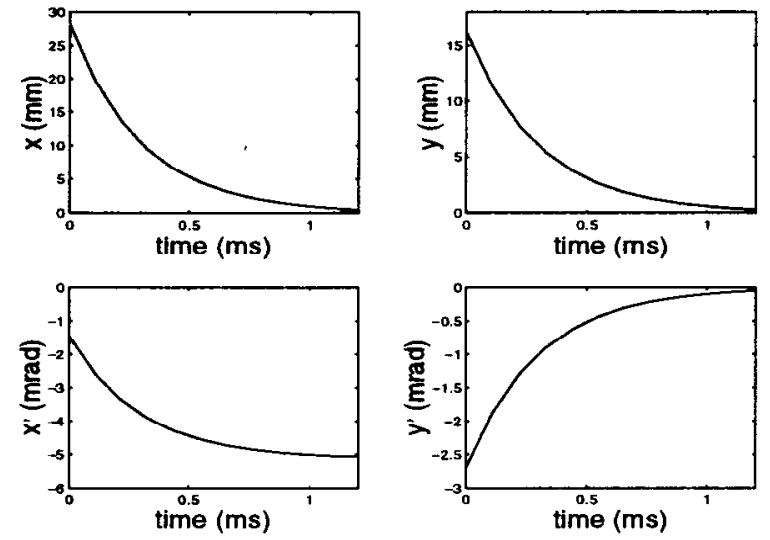

Fig. 2 Orbit bump setting for a $x-y$ correlated painting.

However, such a beam profile is susceptible to transverse coupling due to magnet misalignment and space charge forces, which in turn results in an effective doubling of the maximum emittance in both directions. On the other hand, with the $x-y$ anti-correlated scheme the total transverse emittance is approximately constant during the injection. The resulting oval beam profile achieved by $x-y$ anticorrelated painting, fig. 4 , is immune to the transverse coupling. Table 3 compares the two painting schemes.
Table 3 Comparison of $x-y$ correlated and anti-correlated phase space painting scenarios

\begin{tabular}{|l|c|c|}
\hline painting scenarios & correlated & anti-correlated \\
\hline Foil hitting rate & \multicolumn{2}{|c|}{$1: 2$} \\
\hline Aperture clearance @ inj. & \multicolumn{2}{|c|}{$1: 1.5$} \\
\hline Susceptibility to coupling & yes & no \\
\hline Capability to KV painting & ' no & yes \\
\hline
\end{tabular}

In both painting examples, shown in Fig. 3 and 4, the time constants of the bumps were $0.3 \mathrm{~ms}$ or longer in consideration of eddy current effect in the vacuum chamber. Since painting is adopted in both directions without steering of the injecting beam the minimization of foil hitting and the design of downstream beam line and beam dump for the un-stripped beam become straightforward.
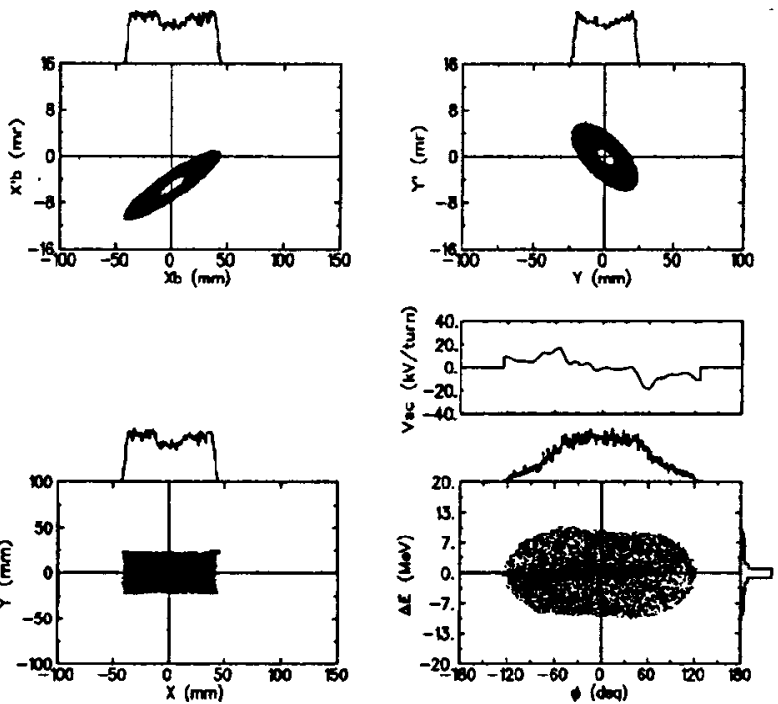

Fig. 3 Phase space distribution at the end of injection achieved by a $x-y$ correlated phase space painting.
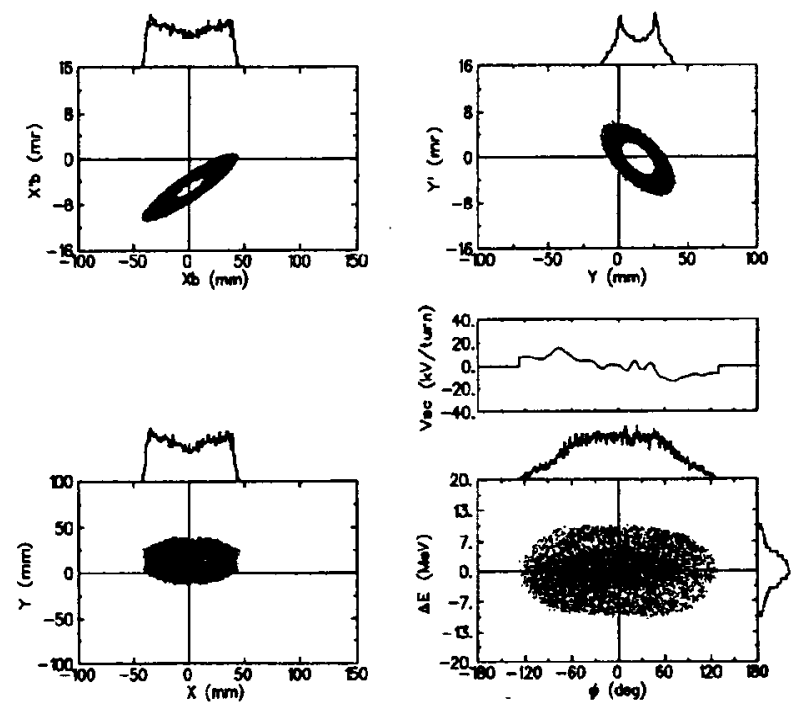

Fig. 4 Phase space distribution at the end of injection achieved by a $x-y$ anti-correlated phase space painting. 


\section{SPACE CHARGE EFFECT}

We compare space charge effects between the beams in the current design of FODO nominal tune lattice and in an alternative design of FODO split tune lattice [12] developed recently as part of design studies in BNL. $10^{5}$ macro particles, with transverse phase space distribution similar to the one shown in Fig. 4 were tracked in the two lattices for 100 turns by SIMPSONS 2D code. Space charge effect in beams with peak current 0-100A, corresponding to the proton accumulation of $0-2 \mathrm{MW}$, was investigated. The rms emittance growth and tune shift due to space charge as functions of peak current are shown in Fig. 5 and Fig. 6 respectively. The lattice functions and space charge effects in $2 \mathrm{MW}$ beams in the two lattices are compared in Table 4 . With the split tune lattice, the vertical beam envelope variation $\left(\beta_{\max } / \beta_{\operatorname{man}}\right)$ is significantly reduced. Correspondingly, the vertical emittance and beam tail/halo generation is also dramatically reduced.

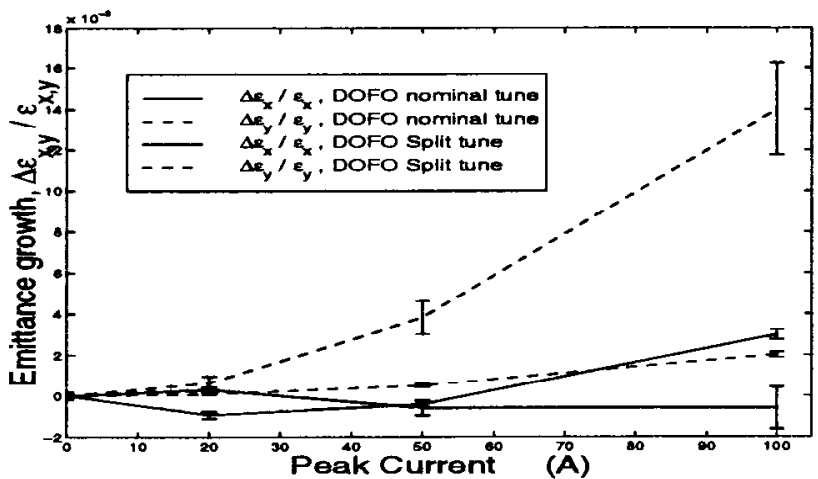

Fig. 5 RMS emittance growth as function of peak current.

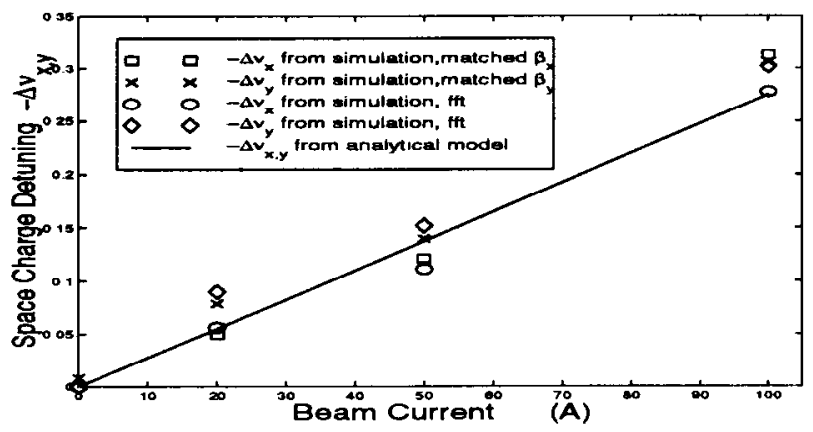

Fig. 6 Maximum space charge tune shifts.

Table 4 Lattice comparison

\begin{tabular}{|c|c|c|}
\hline LATTICE & Unsplit & Split \\
\hline Tune $v,(x / y)$ & $5.82 / 5.80$ & $5.82 / 4.80$ \\
\hline Max. / min. $\beta$-fu & $19.2 / 1.4$ & $19.4 / 2.6$ \\
\hline Max. $\left|D_{x}\right|,(m)$ & 4.0 & 3.9 \\
\hline$\beta_{\mathrm{mu}} / \beta_{\mathrm{mu}}$ & .7 & 7.5 \\
\hline Max. $B_{\text {sp,sD }}$ (at $\left.10 \mathrm{~cm}\right),(\mathrm{kG})$ & 3.0 & 3.2 \\
\hline Max. tune shift, $\Delta v,(x / y)$ & $0.2-0.3$ & $0.2-0.3$ \\
\hline$S C \beta$ growth $\Delta$ & $0.10 / 0.16$ & $0.12 / 0.06$ \\
\hline Emittance growth, & $\sim 0 / .014$ & $.003 / .002$ \\
\hline Tail/halo rate, $x / y\left(10^{-5} /\right.$ turn $)$ & $1.1 / 1.7$ & $1.2 / 0.1$ \\
\hline
\end{tabular}

\section{PSEUDO END TO END SIMULATION}

Driven by searching for suitable painting schemes, injections with various bump settings were simulated in the accumulator ring. Then particle distribution obtain from the ring simulations were tracked to the target through the RTBT line with PARMILA. PARMILA was modified to include scattering effect of a $4 \mathrm{~mm}$ thick inconel window, which is about 2 meter from the target. Then the current distributions are checked against the beam requirement at the target. (Table 1). It was fund that, in a 2-D plan of time constant and bump strength, there is a nice-sized region in which all the bump settings give satisfactory distributions. Fig. 7 shows one example of satisfactory distribution at the target that was achieved by the bump settings shown in Fig. 2. Complete end-toend simulations, from the ion source to the target though linac, HEBT, accumulator ring and RTBT is in progress.

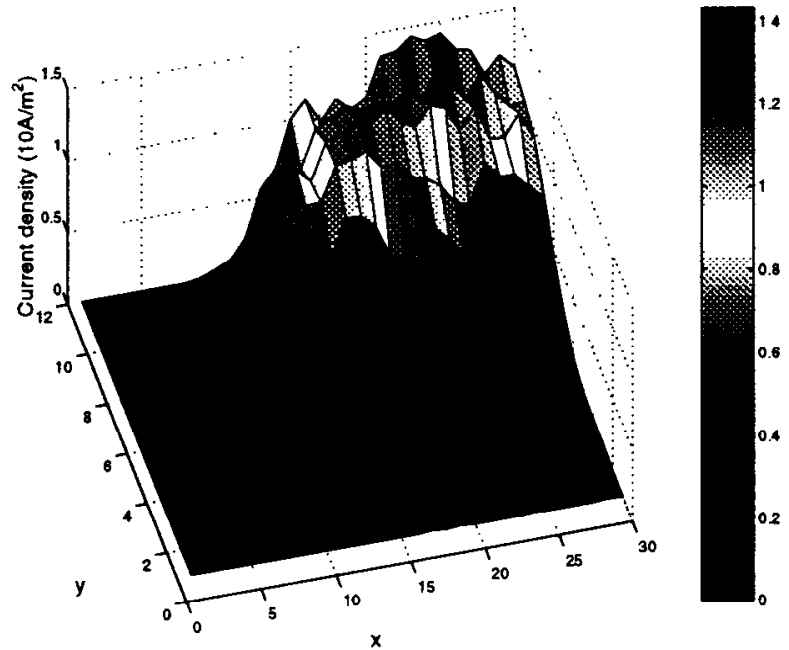

Fig. 7 Current density distribution at the target in units of $10 \mathrm{~A} / \mathrm{m}^{2}$. One quadrant of the beam footprint is shown.

\section{ACKNOWLEDGEMENT}

The authors would like to thank F. Jones and S. Machida for developing ACCSIM and SIMPSONS and making them available. They would also like to thank A. Luccio and N. Malitsky for helping to set-up the codes in BNL.

\section{REFERENCES}

[1] W.T. Weng et al, Proc. of PAC'97, p.972, (1997).

[2] D.Raparia et al, these proceedings.

[3] C.J. Gardner et al, Proc. of PAC'97, p.962, (1997).

[4] D.Raparia et al, Proc. of PAC'97, p.162, (1997).

[5] L.N. Blumberg et al, Proc. of PAC'97, p.159, (1997).

[6] SNS Collaboration, "Spallation Neutron Source Design Manual", p.5.12-1, June 1998.

[7] F. W. Jones, "User's Guide to ACCSIM", TRIUMF Design Note TRI-DN-90-17, June 1990 (and later additions).

[8] S. Machida, "The Simpsons User's Manual", Dallas, 1992.

[9] G. Boicort et al, "Parmila User's Manual", LA-UR-90-127.

[10] N. Malitsky et al, these proceedings.

[11] J. Beebe-Wang, "Study of Longitudinal Injection/stacking in the SNS Accumulator Ring", these proceedings.

[12] C.J. Gardner et al, these proceedings. 